\title{
Space weather: science and effects
}

\author{
Norma B. Crosby \\ Belgian Institute for Space Aeronomy, Ringlaan-3-Avenue Circulaire, Belgium \\ email: norma.crosby@oma.be
}

\begin{abstract}
From the point-of-view of somebody standing outside on a cold winter night looking up at a clear cloudless sky, the space environment seems to be of a peaceful and stable nature. Instead, the opposite is found to be true. In fact the space environment is very dynamic on all spatial and temporal scales, and in some circumstances may have unexpected and hazardous effects on technology and humans both in space and on Earth. In fact the space environment seems to have a weather all of its own - its own "space weather". Our Sun is definitely the driver of our local space weather. Space weather is an interdisciplinary subject covering a vast number of technological, scientific, economic and environmental issues. It is an applicationoriented discipline which addresses the needs of "space weather product" users. It can be truly said that space weather affects everybody, either directly or indirectly. The aim of this paper is to give an overview of what space weather encompasses, emphasizing how solar-terrestrial physics is applied to space weather. Examples of "space weather product" users will be given highlighting those products that we as a civilization are most dependent on.
\end{abstract}

Keywords. Space weather, solar-terrestrial physics, technological and biological effects, forecasting, mitigation

\section{Introduction}

Since the time of the first space missions back in 1957 engineers and operators have been developing methods to mitigate against space weather induced technological problems; and from the beginning of space research the solar-terrestrial physics community has been investigating the physics behing space weather phenomena. In summary, space environment analysis has always existed. However the term "space weather" linking the engineering and the scientific side of the story is more recent. Space weather is an application-oriented discipline and addresses the needs of "users". It is important to note that basic research in the field of solar-terrestrial physics (STP) is necessary for space weather applications. STP relies on basic research and scientific observations, where the product is "scientific". On the other hand space weather is application oriented and relies on continuous monitoring, where the result becomes a "service product". Space weather is indeed a merging of many topics: space science, engineering, medicine, law, etc..

Space weather is defined by the U.S. National Space Weather Program (NSWP) as Conditions on the Sun and in the solar wind, magnetosphere, ionosphere, and thermosphere that can influence the performance and reliability of space-borne and ground-based technological systems and can endanger human life or health", Wright et al. (1993).

A more recent definition of space weather was formulated by members of the COST724 Action, COST724 (2007): "Space weather is the physical and phenomenological state of natural space environments. The associated discipline aims, through observation, monitoring, analysis and modelling, at understanding and predicting the state of the sun, the 
interplanetary and planetary environments, and the solar and non-solar driven perturbations that affect them; and also at forecasting and nowcasting the possible impacts on biological and technological systems."

In more simple terms space weather can be defined as how solar activity may have unwanted effcts on technological systems and human activity. It is a function of our location in the solar system, the behaviour of the Sun and the nature of Earth's magnetic field and atmosphere. Any planet or target in space will have its own local space weather with its own specific characteristics.

Space weather affects everybody either directly or indirectly. So why has progress towards organized efforts to improve the practical solutions to space weather problems become so important lately? There are many good answers to this question: $\underline{1}$. Present society on Earth is deeply dependent on reliable space systems and will be more so in the future (city, rural, and isolated communities) $\underline{2}$. Technical systems are becoming more sensitive to the space environment and will continue so in the future due to developments in technology such as miniaturization $\underline{3}$. STP science has progressed to a stage where the possibilities for useful space weather models and predictions are expected soon and in some cases exist.

Apart from the problems caused to spacecraft by the ultra-high vacuum and extremes of hot and cold in space, spacecraft also have to survive very hostile environments which can severely limit space missions as well as pose threats to humans. Missions need to consider phenomena such as UV, X- and gamma-radiation, energetic charged particles, plasmas and not to forget neutrals (space debris and meteoroids). Fig. 1 illustrates the connection between space weather phenomena and the possible induced space weather effects. This paper offers the reader a short introduction to the fascinating world of space weather. Various of the space environment phenomena are introduced and followed by the potential effects which they can have on technological and biological systems. Thereafter the topic of mitigation is discussed and the paper ends with some final words. For more detailed information about space weather (general reviews) the reader is referred to the excellent space weather books that have been written during this decade Bothmer \& Daglis (2007), Carlowicz \& Lopez (2002), Daglis (2001), Freeman (2001), Hanslmeier (2002), Miroshnichenko (2003), Scherer et al. (2005), Song et al. (2001), Wang \& Xu (2002).

\section{The space environment}

Interplanetary space, better known as the heliosphere can be thought of as a vast magnetic bubble containing the solar system, the solar wind and the interplanetary magnetic field, as well as numerous particle populations, and dust. Without the Sun there would be no life on Earth. However, the Sun is also the origin of phenomena that can influence our daily existence in a more negative way. The Sun's UV is not the only phenomenon that we should worry about here on Earth.

Solar flares, coronal mass ejection, solar wind:

The Sun is our closest star and has a characteristic 11 year cycle. At solar maximum solar activity is largest and at minimum smallest. It is well known that the solar corona is a very dynamic region which is the seat of many phenomena related to magnetic energy releases in a large range of sizes and occurring on time scales going from a few seconds or less to hours. Physical processes leading to magnetic energy releases are analyzed in individual solar flares using multi-wavelength observations and can last from a few minutes to a few hours. The energy released in solar flares varies from $10^{28}$ to $10^{34}$ ergs, 
which is transformed into heating, particle acceleration and mass motions. Solar flares are related to the solar cycle, with their frequency being highest at solar maximum.

Coronographs have allowed us to discover the the world of coronal mass ejections (CMEs). CMEs are huge ejections of plasma in the Sun's outer atmoshere. They are seen as bright features moving outwards through the corona at speeds from 10 to $1000 \mathrm{~km} / \mathrm{s}$ and correspond to massive expulsions of plasma from the solar atmosphere that cause major transient interplanetary disturbances which have significant terrestrial effects. Halo CMEs reaching the Earth interact with Earth's geomagnetic field and are said to be geoeffective. Magnetic storms are one consequence resulting in numerous space weather signatures including the beautiful northern lights. The shock wave driven by a CME has been found to be an excellent particle accelerator (see next sub-section).

As the solar atmosphere is not gravitationally bound to the Sun, there is a flux of ionized matter that escapes continuously from the Sun. It consists largely of ionized hydrogen, contains a weak magnetic field and is significantly influenced by solar activity. The typical speed of the solar wind is $400 \mathrm{~km} / \mathrm{s}$. There exists two regions of the solar wind (high speed and slow speed). The high speed velocity has a velocity that reaches $700 \mathrm{~km} / \mathrm{s}$, while the slow speed wind has a velocity of $300 \mathrm{~km} / \mathrm{s}$. The interaction of high speed and slow speed winds leads to $3-\mathrm{D}$ corotating interaction regions (CIRs) in the heliosphere. Due to enhnaced field strength and rising wind speed within the compression region CIRs can cause magnetic storms.

High speed solar winds mainly occur at the declining phase of solar maximum and have their origin in coronal holes. They are often connected with enhancements in Earth's outer radiation electron belt. This phenomenon is often observed following two weeks of high

\section{Environment}

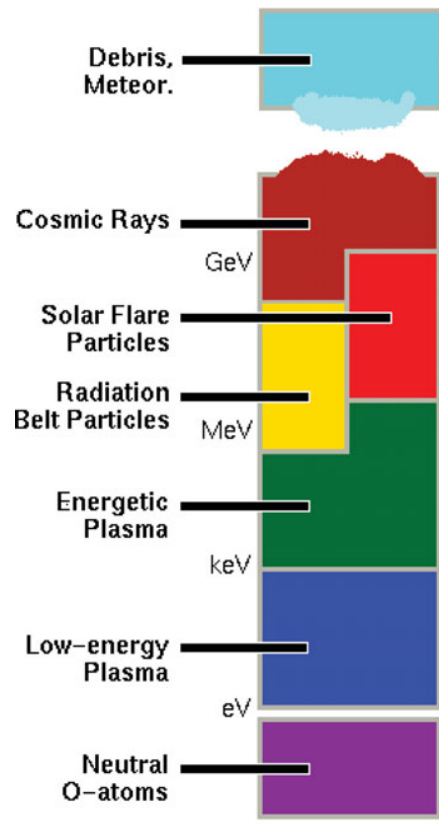

\section{Hazards}
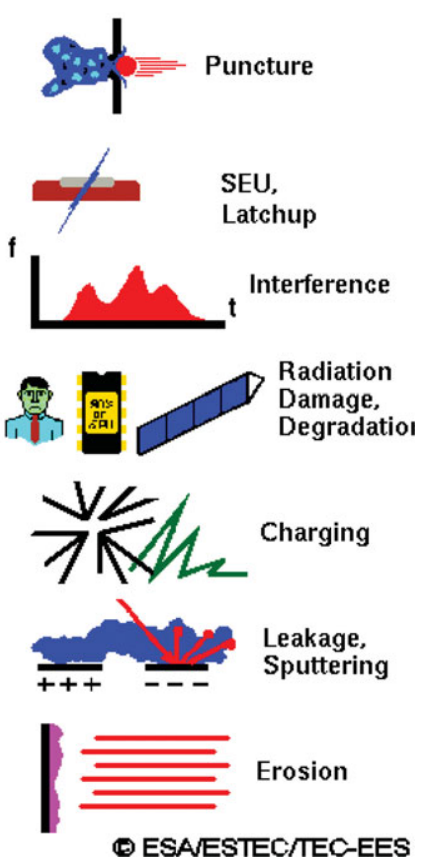

Figure 1. From space environment to space hazards. Courtesy of ESA/ESTEC. 
speed winds. Such events have been associated with a space weather effect coined "deep dielectric charging" which will be discussed later.

Energetic particles:

The most energetic particles (energies up to $10^{21} \mathrm{eV}$ ) found in our solar system, are those that have origins far outside our solar system, namely galactic cosmic rays (GCRs). They are fully ionized particles and their composition is mostly hydrogen nuclei (protons), 7-10\% Helium and $1 \%$ heavier elements. All GCRs are fully ionized, meaning that they consist of nuclei only. As a first approximation the flux of GCRs in near-Earth space can be considered to be isotropic. During solar maximum the increase in the interplanetary magnetic field strength provides enhanced shielding of the heliosphere against penetrating GCR particles. The result is that the GCR population is most intense during solar minimum. On short-term (a few hours) a rapid decrease in GCR intensity may occur following a CME as the magnetic field of the solar wind sweeps away the particles - this is known as a Forbush decrease.

Solar cosmic rays, also called solar energetic particles (SEPs), are associated with a solar flare and/or the shock wave generated by a CME. SEPs are mainly protons, electrons, and $\alpha$-particles with small admixtures of $3 \mathrm{He}$-nuclei and heavier ions up to iron. They are sporadic and difficult to predict, lasting from minutes to days with energies from a dozen of keVs to a few GeVs.

Earth's radiation belts are pricipally composed of naturally occuring energetic charged particles trapped in Earth's inner magnetosphere at equatorial distances ranging from approximately 1.2 to 7 Earth radii. The two doughnut-shaped rings (inner and outer belt) consist mainly of protons (tens $\mathrm{keV}$ - couple of hundreds of $\mathrm{MeV}$ ) and electrons (tens $\mathrm{keV}$ - several $\mathrm{MeV}$ ). These particles are trapped in the Earths magnetic field and their motions in the field consist of: $\underline{1}$. a gyration about field lines, $\underline{2}$. a bouncing motion between the magnetic mirrors found near the Earths poles and $\underline{3}$. a drift motion around Earth.

Table 1 presents these energetic particle populations along with their less energetic counterparts. In summary, these particle populations constitute the major radiation

Table 1. Major particle environments in the heliosphere

\begin{tabular}{|c|c|c|c|}
\hline $\begin{array}{c}\text { Particle } \\
\text { Populations }\end{array}$ & $\begin{array}{l}\text { Energy } \\
\text { Range }\end{array}$ & $\begin{array}{l}\text { Temporal } \\
\text { Range }\end{array}$ & $\begin{array}{l}\text { Spatial Range } \\
\text { (first order) }\end{array}$ \\
\hline Gahatic Cosm ic Rays & $\begin{array}{l}0.1-1000 \mathrm{GeV} \\
\text { (the } 100 \text { to } 1000 \mathrm{M} \mathrm{eV} \\
\text { fluxes consthute the } \\
\text { lergest contrbution) }\end{array}$ & $\begin{array}{l}\text { Conthuous (factor } \\
10 \text { varation w ih } \\
\text { solar cycle) }\end{array}$ & Entire heliosphere \\
\hline $\begin{array}{l}\text { Anom abus Cosm ic } \\
\text { Rays }\end{array}$ & $<100 \mathrm{MeV}$ & Conthuous & Entike heliosphere \\
\hline $\begin{array}{l}\text { Solar Energetic } \\
\text { Particles }\end{array}$ & $\mathrm{keV}-\mathrm{GeV}$ & $\begin{array}{l}\text { Sporadic } \\
\text { (n hutes to days) }\end{array}$ & $\begin{array}{l}\text { Souroe region properties } \\
\text { (flare/CME stes and } \\
\text { evolition) and bound to } \\
\text { CME driven shock }\end{array}$ \\
\hline $\begin{array}{l}\text { Energetic Stom } \\
\text { Particles }\end{array}$ & $\mathrm{keV}-(\triangleright 10 \mathrm{MeV})$ & Hours-Day & Bound to shock \\
\hline $\begin{array}{l}\text { Corotating Interaction } \\
\text { Regibns }\end{array}$ & $\mathrm{keV}-\mathrm{MeV}$ & $\begin{array}{l}\text { Few days } \\
\text { (recument) }\end{array}$ & $\begin{array}{c}\text { Bound to CIR shock and } \\
\text { com pression region }\end{array}$ \\
\hline $\begin{array}{l}\text { Particles accelerated } \\
\text { atphnetary bow } \\
\text { shocks }\end{array}$ & $\mathrm{keV}-\mathrm{MeV}$ & Conthuous & Bound to bow shock \\
\hline $\begin{array}{l}\text { Trapped Particl } \\
\text { Popultitions }\end{array}$ & $\begin{array}{l}\text { Tens keV - couple of } \\
\text { hundreds of MeV } \\
\text { (fiprprotons) } \\
\text { Tens keV - severalM eV } \\
\text { (fior electrons) }\end{array}$ & $\begin{array}{l}\text { Variations } \\
\text { "m nutes-years" }\end{array}$ & $\begin{array}{l}\text { Variations } \\
\text { "heightw idth" }\end{array}$ \\
\hline
\end{tabular}


environments in the heliosphere (for a detailed overview see Crosby in Bothmer \& Daglis (2007)).

\section{Neutrals (particulates):}

Particulates include meteoroids, space debris and dust. Meteoroids are particulates in space of natural origin (nearly all of them originate from asteroids or comets). Space debris, on the other hand, is man-made. Dust is a term used for particulates which have a direct relation to a specific solar system body and which are usually found close to the surface of this body (e.g., lunar, Martian or cometary dust). Particulates are not space weather effects, but they are affected by space weather. They can influence spacecraft engineering and operations. The damage caused by collisions is a function of the size, density, and speed distribution of the impacting particles, and also depends on the shielding of the spacecraft.

\section{Space weather technological induced effects}

Technical users of space weather products include all those technologies that depend on spacecraft and airline reliability, as well as electrical and oil companies. Examples of "technological systems" that encounter space weather induced problems include the electrical networks for power transmission, radio communications, space-borne synthetic aperture radars, global positioning and navigation systems using satellites, geomagnetic surveys, and pipelines (corrosion effects). Satellites monitoring the Earth (e.g., remote sensing) and those used for security purposes may be affected too. It is vital that satellites function for the monitoring of natural catastrophes (e.g., forest fires, floods, avalanches, etc.) or during military operations (e.g., radio communications relying on the ionosphere, localization techniques), so that wrong information is not transmitted, or that system performance is not degraded. The space hazards that a spacecraft may encounter are a function of its orbit, i.e. the various space environment phenomena can affect satellites or spacecraft having different orbits differently.

Auroras are the oldest known manifestation of space weather effects at planet Earth and are due to energetic electrons spiralling down the Earth's magnetic field lines towards the polar regions and striking the upper atmosphere (oxygen atoms or nitrogen molecules). Energy from an atom or molecule excited by a fast electron is released as a photon; different colours of auroral lights are emitted. On the other hand Barlow (1849) was the first person to publish systematic observations of spontaneous electrical currents observed in the wires of an electric telegraph. Since then the effects of space weather on space- and ground-based systems have grown due to our technological developments. The chronological order of the various discoveries of space weather effects is illustrated in Fig. 2.

Radiation effects:

Radiation damage to on-board electronics may be separated into two categories: 1 . Single Event Effects (SEEs), ․․ Cumulative Radiation Damage. SEEs are individual events which occur when a single incident ionizing particle deposits enough energy to cause an effect in a device (e.g. Upset, Latchup, Burnout, ). This effect is not only observed onboard spacecraft, but has also been encountered on airplanes. Presently, airplanes fly in average at heights between 9,000 and 12,000 $\mathrm{m}$ where one finds both primary radiation (protons, electrons), and secondary radiation (neutrons, mesons and electrons). Neutrons are the most dangerous as they have high penetration power because they are electrically neutral. This is the reason why it is estimated that at such altitudes radiation hazards are comparable to those on satellites situated in LEO (Koskinen, 2001). 
Cumulative Radiation Damage is divided into two types: Total Ionizing Dose (TID) and Total Non-Ionizing Dose (also known as "displacement damage dose"). TID occurs when radiation penetrates the constituents of electronic components and the lost energy is stored in the material. Displacement effects are caused by an atom displacement from the normal lattice position to an adjacent position, thus creating structure damage and resulting in recombining centers that deteriorate the electrical characteristics of the material. Energetic particles degrade the semiconductor material and reduce the expected lifetime over which for example solar cells are able to produce energy for the spacecraft.

Electrostatic discharges:

Electrostatic charging occurs on satellites crossing or situated in the near-Earth space environment and is classified into two main categories: Surface Charhing and Internal Charging (also known as "deep dielectric charging"). Surface charging is due to lowenergy electrons in the plasma with energies ranging from several $\mathrm{keV}$ to several tens of $\mathrm{keV}$ and occurs, first of all because of the different mobility characteristics of the charges that constitute the near-Earth plasma. The plasma is energetically neutral. However, electrons are much more mobile (flux of electrons to an "uncharged" surface normally exceeds the flux of ions) and the net effect is that surfaces charge negatively with respect to the local plasma. Deep dielectric charging occurs if electrons have energies higher than $100 \mathrm{keV}$. These high-energy electrons can penetrate the outer shielding of a satellite and deposit charges in the dielectric materials inside the satellite.

Electrical transients from surface discharging or internal charging can masquerade as "phantom commands" appearing to spacecraft systems as directions from the ground. This can result in loss of control of instruments and power or propulsion systems.

Atmospheric drag:

A denser atmosphere causes more drag. Objects in Low Earth Orbit (LEO) encounter atmospheric drag in the form of gases in the thermosphere (approximately $80-500 \mathrm{~km} \mathrm{up}$ ) or exosphere ( $\approx 500 \mathrm{~km}$ and up), depending on orbit height. The LEO altitude is usually not less than $300 \mathrm{~km}$ because that would be impractical due to the larger atmospheric drag. Emissions from the Sun (including, the highly variable X-ray and ultraviolet output)

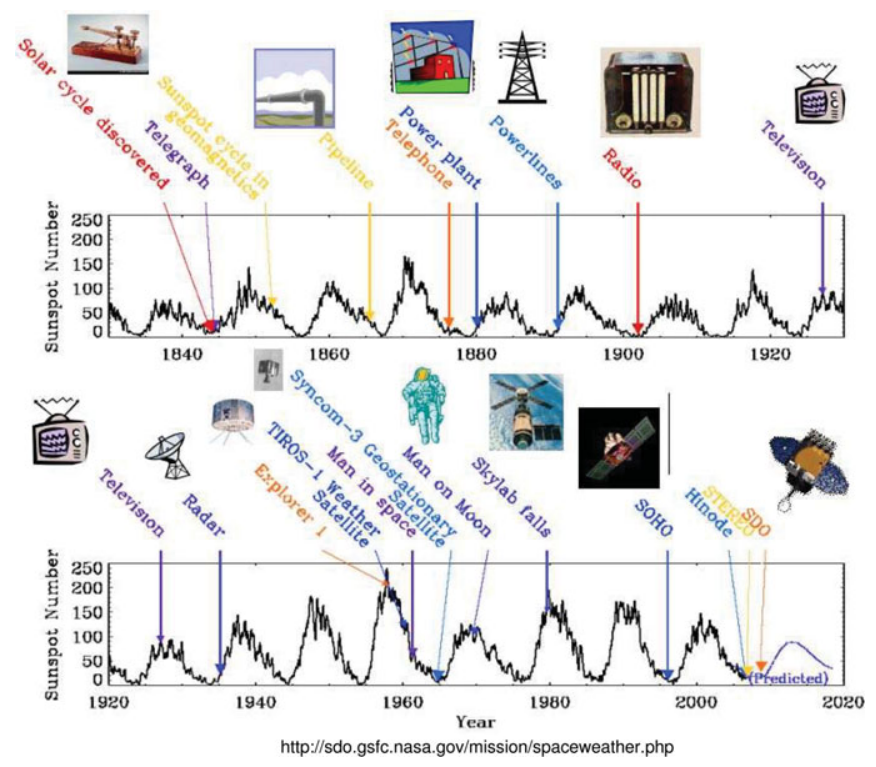

Figure 2. Time line of the discovery of space weather effects. Courtesy of NASA/GSFC. 
cause the upper atmosphere to heat and expand. If magnetic activity is also triggered in the Earths magnetosphere, intense electric currents flow through the ionosphere and upper atmosphere, and the energy deposited at high latitudes increases the heating and expansion of the atmosphere in these regions markedly.

The most famous example of this effect was Skylab, which burned up in the atmosphere on July 11, 1979 after its orbit deteriorated for 5 years. Some satellites (e.g. Compton Gamma Ray Observatory), have onboard jets to compensate for orbit decay. The Hubble Space Telescope (HST) has no jets or engines of any kind for propulsion, so the only way to restore the altitude is to grab it and move it ["space shuttle during HST servicing missions"]. The drag effect has its beneficial side too and this concerns the problem of space debris. When debris falls towards Earth it burns up in the atmosphere. This is a natural occurring procedure to reduce the number of cosmic debris.

\section{Radio communications:}

Earth's ionosphere, created by the ionization of the upper atmosphere by solar EUV and X-ray radiation, plays an important role in radio communications. Spatial scales of inhomogeneities in the ionosphere vary from thousands of kilometres to turbulence with scale sizes of less than a meter. Likewise, the temporal scales vary over many orders of magnitude from many years (solar cycle effects on ionospheric propagation) to hours or even minutes (the scale of weather phenomena). High frequencies (HF) are attenuated in the $\mathrm{D}$ layer of the ionosphere and then reflected in the $\mathrm{F}$ layer. HF propagation anomalies are due to ionospheric changes resulting from solar flares, proton events and geomagntic storms.

Because of solar flares, the increased intensity of the EUV radiation may result in the increase of the ionization level of the D layer, where the attenuation of the HF waves increases and produces Short Wave Fadeouts (SWF). The attenuation can be so high that it may lead to the interruption of radio transmission. SWFs is a particular ionospheric solar flare effect under the broad category of sudden ionospheric disturbances.

Very High Frequencies (VHF) and Extremely High Frequencies (EHF) are not reflected by the ionosphere. They cross the ionosphere where they are subject to a refraction phenomenon. These frequencies are used to communicate with artificial satellites for command transmission and data reception. At times of more intense ionization and increased concentration and temperature in the ionosphere a phenomenon known as "plasma bubbles" will be created. When radio waves go through such irregularities, the wave intensity and propagation speed are attenuated and changed, respectively. The result is signal fading or data downlink (attenuation of the signal and loss of data).

The ionosphere in polar regions can be badly affected by high energy solar protons (>10 MeV) arriving hours to days after the solar disturbance (e.g. strong solar flare). Very strong ionisation of the D-region leads to absorption of HF signals similar to an HF Fadeout. This is called a Polar Cap Absorption (PCA) event. PCAs can last for several days following a large solar flare in contrast to a low/mid latitude fadeout which generally lasts just an hour or two.

Ground effects:

Geomagnetically Induced Currents (GICs) are the ground end of the space weather chain. A GIC is defined as a current connected with a geomagnetic variation and a manmade conductor (e.g. power transmission systems, oil and gas pipelines, telecommunication cables, railways). Space weather storms produce intense and rapidly varying currents in the magnetosphere and ionosphere which cause time-dependent magnetic fields seen as geomagnetic disturbances or storms. As expressed by Faradays law of induction, a time variation of the magnetic field is always accompanied by an electric field. The geomagnetic disturbance and the geo-electric field observed at the Earth's surface not only 
depend on the "primary" space currents, but are also affected by "secondary" currents driven by the electric field within the conducting Earth. In particular for the electric field, the secondary contribution is essential. The horizontal geoelectric field drives ohmic currents (GICs) in technological conductor networks (see Fig.3). Currents induced in power lines flow to ground through substation transformers. Here they cause saturation of the transformer core which can lead to a variety of problems. Increased heating has caused transformers to burn out. Also extra harmonics generated in the transformer produce unwanted relay operations, suddenly tripping out power lines. The stability of the whole system can also be affected as compensators switch out of service. Such a sequence of events led to the Quebec blackout of 13 March 1989.

Biological effects:

High frequency radiation or high-energy particles can knock electrons free from molecules that make up a cell. These molecules with missing electrons are called ions, and their presence disrupts the normal functioning of the cell. Cells that reproduce rapidly (skin, eyes, blood-forming organs) are the most susceptible to damage because they cannot repair themselves easily while replicating. The most severe damage to the cell results when the DeoxyriboNucleic Acid (DNA) is injured. DNA is at the heart of the cell and contains all the instructions for producing new cells. Symptoms of radiation sickness are severe burns that are slow to heal, sterilization, cancer and other damages to organs. Mutations or changes in the DNA can be passed along to offspring.

These above listed biological effects include those associated with current and future astronauts and cosmonauts. However, aircraft crew and passengers onboard airlines are also under risk of radiation, especially on polar routes. In this region of the geomagnetic field, the magnetic field lines are open and energetic particles can reach down to lower altitudes. The Council of the European Union adopted Directive 96/29 Euratom on 13 May 1996. Article 42 of the Directive imposes requirements relating to the assessment

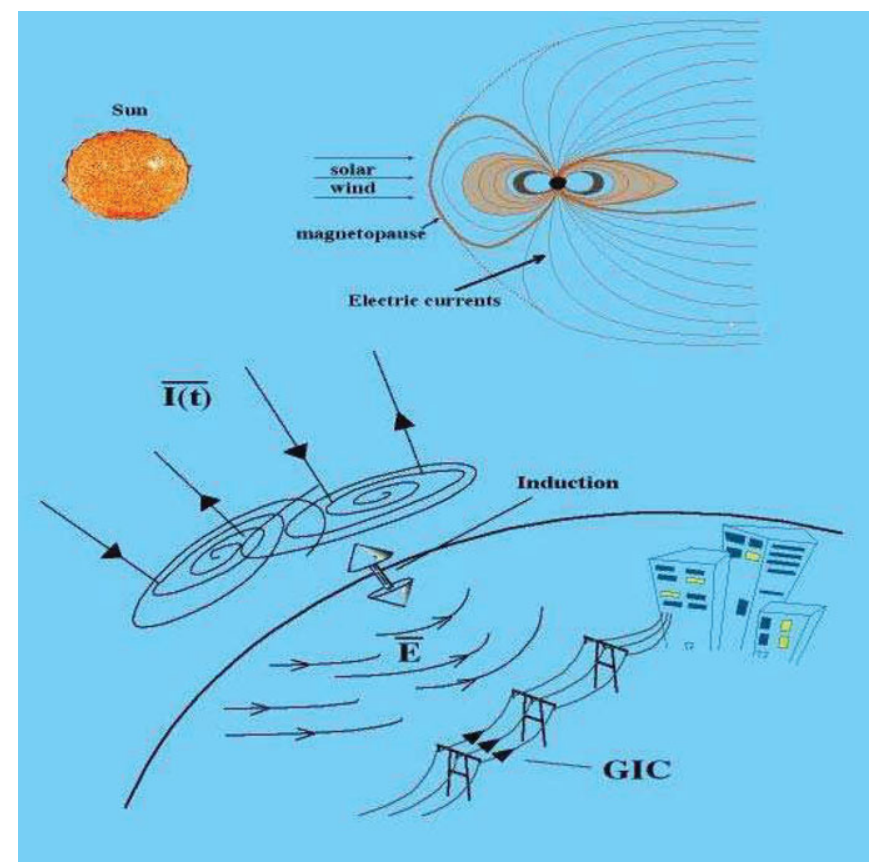

Figure 3. Schematic drawing of the creation of geomagtically induced currents. Courtesy of Finnish Meteorological Institute. 
and limitation of air crew members exposure to cosmic radiation and the provision of information on the effect of cosmic radiation.

During the famous Octber-November 2003 solar events all commercial aviation interests were made aware of the radiation storm levels on October 28 to 29, when the Federal Aviation Administration (FAA) issued its first ever advisory suggesting that flights travelling North and South of 35 degrees latitude were subject to excessive radiation doses.

\section{Mitigation}

Health risks for long duration interplanetary explorative missions and those encountered so far in manned space flight differ significantly in two major features: 1 . "Emergency returns" are ruled out. 2. The loss of geomagnetic shielding available in low Earth orbit with an associated non-negligible risk for acute early radiation diseases.

There are two approaches in tackling space weather and its unwanted effects. First there is the classical engineering approach which relies on shielding, mitigation for electronics and charging, mitigation in radio communications and medical mitigation. Spacecraft "age" through continual bombardment by energetic particles. The key to radiation protection is the understanding of the space environment and its interaction with shielding. An important issue concerning shielding is the problem of secondary radiation in materials. New forms of shielding materials are imagined and more impetus should be placed on polymer research in regard to the development of resistant light atomic weight shielding. Ofcourse the faster the trip the better, i.e. development of innovative transportation technologies and new propulsion systems as well as orbit optimization, are highly important if not the most important challenges. The ultimate goal is to minimize radiation together with all other health effects and technical hazards by optimizing orbit parameters and shielding. Maris \& Crosby (2008) is a review paper concerning the technical effects induced by space weather as well as the techniques used for mitigation.

The second approach in tackling space weather relies on avoiding the phenomena that may cause these effects in the first place, respectively the prevention approach "space weather forecasting". This can only happen if one has a reliable description of the space environment that the spacecraft will encounter? This relies on real-time measurements (space-borne and on Earth) and models of the various environments? In this way one can monitor the space weather and warn potential space weather customers of "bad weather". In the future it will be the users of space weather forecasting centers that must set the requirements for the time scales and precision of the predictions.

\section{Final words}

Humans and technology (in space and on Earth) are vulnerable to the space weather. Some of the effects can be corrected for and/or mitigated against, others not. Modern society is increasingly becoming dependent on the usage of artificial satellites and will be more so in the future. In summary, scientists, engineers, medical doctors, etc. from many disciplines must learn to work together to optimize our understanding of the space environment and its interaction with our daily lives.

In addition there will be many opportunities in regard to space weather in the near future (Missions to other planets and moons [manned and robotic], colonies on other planets such as Mars, Mining on other planets, moons, asteroids and terra-forming, transportation technology, space tourism, space hotels, emergence of space entrepreneurs). Indeed the field of space weather has a bright future both from the scientific as well as the techniological point-of-view. 


\section{References}

Bothmer, V. \& Daglis, I. A. (eds) 2007, in: Space Weather: Physics and Effects, Springer Praxis Books

Carlowicz \& Lopez 2002, Storms from the Sun: The Emerging Science of Space Weather, National Academies Press

Cost724 2007 Cost724 Final Report

Daglis, I. A. 2001 Space Storms and Space Weather Hazards, Kluwer Academic Publishers

Freeman, J. W. 2001 Storms in Space, Cambridge University Press

Hanslmeier, A. 2002 Kluwer Academic Publishers

Koskinen, H., Tankanen, E., Pirjola, R., Pulkkinen, A., Dyer, C., Rodgers, D., Cannon, P., Mandeville, J. C. \& Boscher, D. 2001, Space Weather Effects Catalogue, ESWS-FMI-RP0001

Maris, O. \& Crosby N. 2008, in: G. Maris \& M. D. Popescu (eds.), Research SignPost Ed. House, India (In print)

Miroshnichenko, L. 2003, Radiation Hazard in Space (Kluwer Academic Publishers)

Scherer, K. et al. 2001, in: Scherer, K., Fichtner, H., Heber, B. \& Mall, U. (eds.), Behind a Slogan (Lecture Notes in Physics)

Song, P. et al. (eds) 2001, in: Space Weather (American Geophysical Union)

Wang, H. \& Xu, R. (eds) 2002, in: Proceedings of the COSPAR Colloquium on Solar-Terrestrial Magnetic Activity and Space Environment (Elsevier Science Ltd.)

Wright, Jr., J. M., Lennon, T. J., Corell, R. W., Ostenso, N. A., Huntress, Jr., W. T., Devine, J. F., Crowley, P., \& Harrison, J. B. 1995, The National Space Weather Program, The Strategic Plan., Office of the Federal Coordinator for Meteorological Services and Supporting Research, FCM-P30-1995, Washington DC, USA, August

\section{Discussion}

VLAHOS: Can we make progress with the use of many different codes interacting since we are dealing with a "system" of interacting elements?

Crosby: There are efforts in trying to connect the different regions (solar atmosphere, magnetosphere, ionosphere/atmosphere) of the Sun-Earth connection. For example, the goal of the Center for Integrated Space Weather Modeling (CISM) is to create a physicsbased numerical simulation model that describes the space environment from the Sun to the Earth.

Grebowsky: You just briefly discussed radiation belt physics, but it is the prime source of the spacecraft charging effects and spacecraft failures. The one high energy outer belt enhancement mentioned has been attributed actually to an enhanced magnetosphere E-field induced. NASA's RB storm probe mission is geared to solve these problems. Unfortunately there are no ionospheric missions planned. 